\title{
A Multiantenna Approach to Maximize Wireless Power Transfered to Implantable Devices
}

\author{
H. Dinis, I. Colmiais, P. M. Mendes \\ CMEMS, University of Minho, Guimarães, Portugal, hugodcdinis@gmail.com
}

\begin{abstract}
Power delivery from an external source to implanted devices through wireless links is hindered by SAR regulations, which limit the amount of power radiated by an external source that reaches an implant. In order to increase the power delivered to the implant, we studied a solution with two transmitters and compared results with the common single transmitter link. HFSS and COMSOL have been used to show that a power increase of around $40 \%$ inside a human head model was achieved while respecting SAR limits and keeping tissue temperature under control, stabilizing at around $37.4{ }^{\circ} \mathrm{C}$.
\end{abstract}

Keywords-Wireless power transfer, SAR, Implanted neurodevice

\section{INTRODUCTION}

Implantable medical devices are becoming smaller and more efficient, requiring less power to achieve their purpose. In order to allow further miniaturization, the cumbersome batteries that add to (and often limit) the device's final volume must be reduced in size or even completely removed. For this to be possible, the devices must be powered through an external source.

Currently, Wireless Power Transfer (WPT) links are developed resorting to two techniques: maximizing the receiver's efficiency and/or positioning the receiver with an orientation that favours energy transmission efficiency [2]. Solutions capable of delivering hundreds of $\mathrm{mW}$ to the implants have resulted from this approach, but the device's dimensions were in the order of the centimetre $[3,4]$. When considering solutions in the millimetre scale, only a few devices that can deliver a couple of $\mathrm{mW}$ have been reported, with most of them operating in the $\mu \mathrm{W}$ region $[5,6]$. There has been little work on the improvement of the delivered power through the manipulation of other parameters such as the number of transmitters, which is already done in other fields [7]. SAR levels are influenced by the tissue's dielectric properties, geometry, dimensions and orientation, and by the incident wave's frequency and modulation [8]. Therefore, we believe that instead of focusing on the optimization of the link between a receiver and a transmitter in a given position and with a set frequency and polarization, the possibility of using a multidimensional approach should be explored.

In this work, we implemented a solution with two transmission antennas which, when compared with the single transmitter solution, increased energy availability inside a simplified human head model, while maintaining the same SAR levels.

\section{PROBLEM SETUP}

Fine and detailed electromagnetic simulation results related to brain structures are possible, but may require complex and time consuming simulation setups [9]. Moreover, simulation of interaction between electromagnetic waves and biological structures requires tools with multiphysics capabilities. In this work, simulations were done using low computational power, which led to the decision of using two simulation tools (HFSS and COMSOL) with a simplified head model. HFSS allows for the quick analysis of the electromagnetic problem and to quickly arrive to a preferred antenna configuration. COMSOL, on the other hand, allows the inclusion of head thermal regulation and quickly allows the study of temperature maps for the obtained antenna configurations.

Both simulation tools were used to simulate the same simplified head model's interaction with one and two dipole antennas radiating power at $1 \mathrm{GHz}$, with antennas being placed at $300 \mathrm{~mm}$ from the centre of the model, as demonstrated in Fig. 1. This step was implemented to validate both tools' results. The E-field inside the brain was calculated on both software, using a head model consisting of a $45 \mathrm{~mm}$ radius sphere, representing the brain, enveloped in two layers, corresponding to skin ( $1 \mathrm{~mm}$ thick) and skull (4.1 mm thick), all defined with the corresponding tissue's dielectric properties for $1 \mathrm{GHz}$. Fig. 2 presents slices from the results of both simulations across the XZ plane, where the E-field shapes match in both software. Furthermore, peak values, despite inherent software discretization, are very similar. HFSS presented a peak E-field of $92 \mathrm{~V} / \mathrm{m}$ for one antenna and $114 \mathrm{~V} / \mathrm{m}$ for the two antenna scenario, while COMSOL computed $100 \mathrm{~V} / \mathrm{m}$ and $110 \mathrm{~V} / \mathrm{m}$ for the same scenarios.

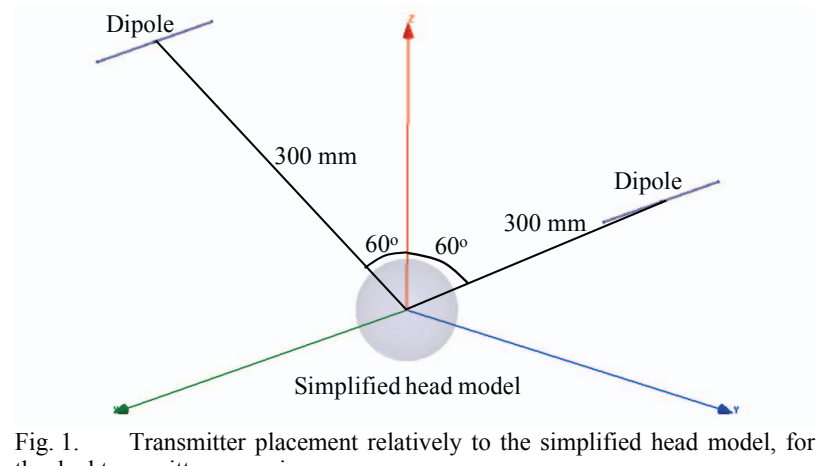
the dual transmitter scenario. 

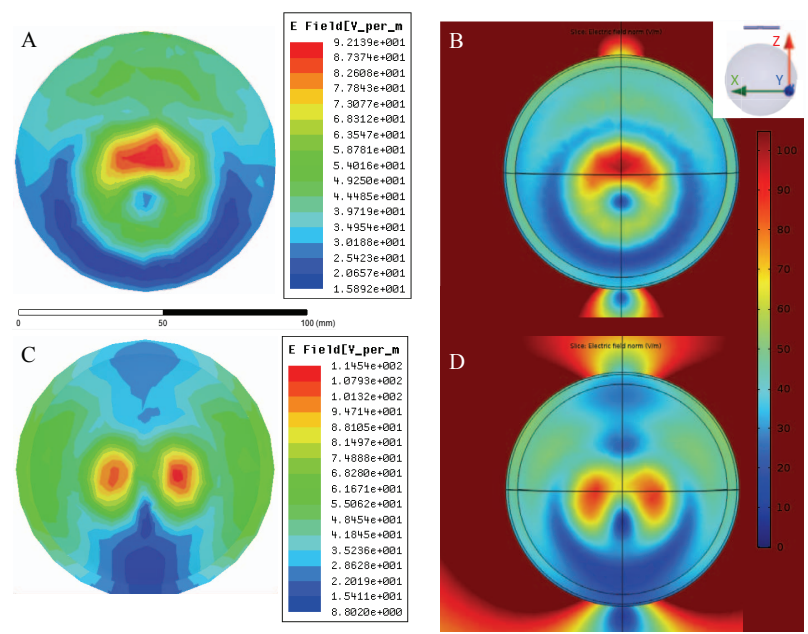

Fig. 2. E-field magnitude in the XZ plane for one antenna: A - HFSS B - COMSOL; and two antennas: C - HFSS, D - COMSOL.

\section{RESUlTS AND DISCUSSION}

Simulations were used to trim a WPT solution based on two antenna solution, without surpassing SAR limits, and maintaining tissue temperature under control. In HFSS, the dipoles' positions, power input and phase were designed by simulation to maximize the Poynting vector's value inside the brain, while keeping the $10 \mathrm{~g}$ averaged SAR below the $2 \mathrm{~W} / \mathrm{kg}$ established limit. When one dipole was used, SAR limits its power to $21 \mathrm{~W}$. In the two dipole model, SAR limits each dipole power to $35.5 \mathrm{~W}$, where the combination yielding the highest Poynting was with dipoles placed at $60^{\circ}$ from the vertical axis, with phases set to $0^{\circ}$ and $210^{\circ}$. Fig. 3 displays the Poynting vectors' magnitude for a slice that contained the hotspot. The usage of two antennas increased the available power from $55 \mathrm{~W} / \mathrm{m}^{2}$ to $76 \mathrm{~W} / \mathrm{m}^{2}$ in the hotspot, an increase of around $40 \%$, which is also noticed in the surrounding areas.

With the previous parameters inserted into the COMSOL models, temperature values were obtained. The model was configured with the tissues' thermal properties, such as blood perfusion, metabolic heat generation rate, thermal capacity and thermal conductivity. Fig 4 contains the results from the one and two dipole cases. With no external radiation source, the model's temperature stabilizes at around $37.3{ }^{\circ} \mathrm{C}$. When stimulated by one or two antennas, it increases to around $37.4{ }^{\circ} \mathrm{C}$, a very small variation. Moreover, the maximum temperature in both scenarios is almost identical $\left(37.42{ }^{\circ} \mathrm{C}\right.$ and $37.46^{\circ} \mathrm{C}$, respectively), which was expected as SAR was the same for both cases.

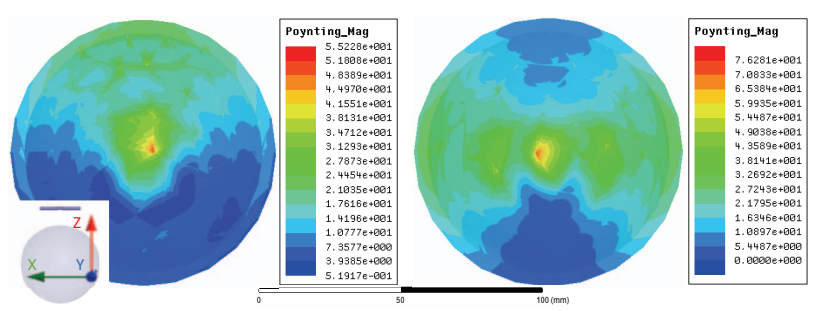

Fig. 3. Poynting vector magnitude in the hotspot in a slice parallel do the $\mathrm{XZ}$ plane for one antenna (left) and two antennas (right).

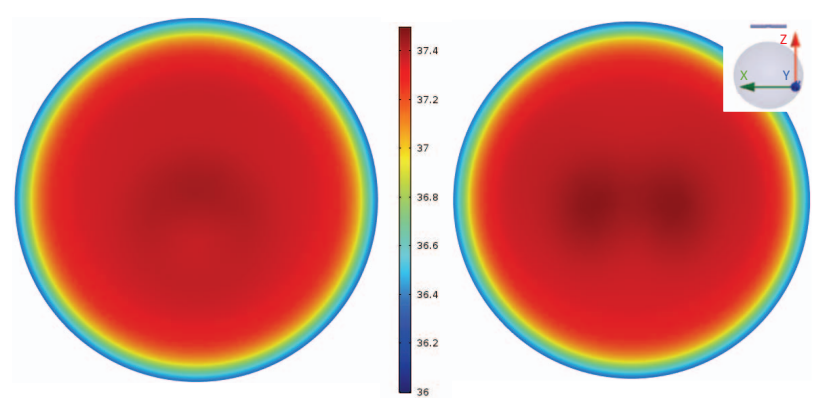

Fig. 4. Temperature plot resulting from the interaction of the head model with one (left) and two (right) antennas.

\section{CONCLUSION}

With this work, it has been demonstrated that adopting a multidimensional approach to the design of WPT links might yield significant improvements over current methods. More specifically, it has been shown that using two transmission antennas instead of one, for the same SAR and temperature levels, the available power inside the head may increase by around $40 \%$. As future work, more directive transmission antennas will be used, as we believe that higher Poynting vector values might be achieved and that it will allow us to accurately define the position of the hotspot inside the human head by adjusting the antennas' relative position and phase.

\section{ACKNOWLEDGMENTS}

Work supported by FCT under project PTDC/EEITEL/5250/2014, by FEDER funds through Projeto 3599 Promover a Produção Científica e Desenvolvimento Tecnológico e a Constituição de Redes Temáticas (3599PPCDT).

\section{REFERENCES}

[1] E. Katz, Implantable Bioelectronics. 2014

[2] B. J. Walk, S. M. Ieee, J. Weber, S. M. Ieee, C. Soell, R. Weigel, F. Ieee, G. Fischer, S. M. Ieee, T. Ussmueller, and S. M. Ieee, "Implants for Telemonitoring," vol. 102, no. 11, pp. 1811-1832, 2014.

[3] D. Borton, M. Yin, J. Aceros, and A. Nurmikko, "An implantable wireless neural interface for recording cortical circuit dynamics in moving primates," J. Neural Eng., vol. 10, no. 2, p. 26010, 2013.

[4] C. Mestais, G. Charvet, F. Sauter-starace, M. Foerster, and D. Ratel, "WIMAGINE : Wireless 64-Channel ECoG Recording Implant for Long Term Clinical Applications," vol. 23, no. 1, pp. 10-21, 2015.

[5] P. Troyk et al, "In-Vivo Tests of a 16-Channel Implantable Wireless Neural Stimulator *," vol. 4, pp. 22-24, 2015.

[6] R. Muller et al, "A Minimally Invasive 64-Channel Wireless ECoG Implant," vol. 50, no. 1, pp. 1-16, 2015.

[7] N. Pan, A. S. Boaventura, M. Rajabi, D. Schreurs, N. B. Carvalho, and S. Pollin, "Amplitude and Frequency Analysis of Multi-sine Wireless Power Transfer," Integr. Nonlinear Microw. Millimetre-wave Circuits Work. (INMMiC), 2015, no. 1, pp. 1-3, 2015.

[8] C. K. Chou et al, "Radio frequency electromagnetic exposure: tutorial review on experimental dosimetry", Bioelectromagnetics, vol. 17, no. 3, pp. 195-208, 1996.

[9] L. Zhao, Q. Ye, K. Wu, G. Chen, and W. Yu, "A New High-Resolution Electromagnetic Human Head Model," IEEE Antennas and Propagation, Vol 56 No. 5, October, 2016. 